\title{
VISCOELASTIC RAYLEIGH WAVES II*
}

\author{
BY \\ P. K. CURRIE (University College, Dublin) \\ AND \\ P. M. O'LEARY (College of Technology, Dublin)**
}

\begin{abstract}
The number of possible surface waves on a viscoelastic half-space is investigated numerically as a function of the material parameters. The results are applied to simple theoretical models of viscoelastic behavior and also to experimental data. It is concluded that two surface waves are possible on some rocks and on nearly all solid polymers.
\end{abstract}

1. Introduction. In an earlier paper [1] a general analysis was given of the propagation of surface waves over a half-space of homogeneous, isotropic, linearly-viscoelastic material. It was found in particular that (in contrast with elastic materials):

(i) more than one surface wave may be possible;

(ii) the waves may be either direct or retrograde at the surface;

(iii) the sense of the motion may change at many or no levels below the surface:

(iv) the surface wave speed may be greater than the body wave speeds.

Our purpose in this paper is to continue the discussion of property (i). In the earlier paper [1], it was shown by means of specific examples that more than one surface wave may be possible for certain values of the complex Lamé moduli $\lambda, \mu$. Here in Secs. 3 and 4 we investigate systematically the number of surface waves that are possible for given values of $\lambda$ and $\mu$. For the most part numerical methods are used, but some analytical results are deduced. The results indicate that calculation will normally be required to determine the number of possible surface waves for a given material at a given frequency. A priori, there seems no reason to suppose that there will be one rather than two waves. We have found no case of more than two waves.

In Sec. 4 we apply our results to two simple models for viscoelastic behavior, the Kelvin-Voigt and Maxwell models, and show how the number of roots varies with frequency. Finally in Sec. 5 we quote experimental data which indicate that two surface waves are possible at certain frequencies on granite and that on solid polymers there are typically two surface waves.

2. Basic equations. We quote the few equations from [1] that are needed subsequently, using the notation of that paper.

A Rayleigh wave propagating over the free surface $x_{3}=0$ of a half-space of homogeneous, isotropic, linearly-viscoelastic material will have a displacement of the form [1, Eq.

* Received June 21, 1977. The authors are grateful to Prof. M. Hayes for help and suggestions.

** Present address: University College, Cork. 
$(3.1)]$

$$
u_{i}=\sum_{N=1}^{2} U_{i}{ }^{N} \exp i \omega\left(s_{p}{ }^{(N)} x_{p}-t\right)
$$

where $\omega$ is the frequency of the wave. The slownesses $s_{i}{ }^{11}, s_{i}{ }^{2}$ satisfy [1, Eqs. (3.3), (3.4)]

$$
\begin{gathered}
s_{1}{ }^{1}=s_{1}{ }^{2}=s_{1}, \quad s_{2}{ }^{(1)}=s_{2}{ }^{(2)}=0, \\
{\left[s_{3}{ }^{1}{ }^{1}\right]^{2}=(\rho / \mu)-s_{1}{ }^{2}, \quad\left[s_{3}{ }^{2}\right]^{2}=(\rho /[\lambda+2 \mu])-s_{1}{ }^{2},}
\end{gathered}
$$

where $x_{1}$ is the direction of propagation of the wave. $\lambda$ and $\mu$ are the complex Lamé moduli, which are functions of the frequency $\omega$. They satisfy the following conditions:

$$
\begin{array}{ll}
\mu^{+}>0, & 3 \lambda^{+}+2 \mu^{+}>0, \\
\mu^{-} \leq 0, & 3 \lambda+2 \mu^{-} \leq 0 .
\end{array}
$$

where the superscripts + and - denote the real and imaginary parts respectively of complex numbers or vectors. The coefficients $U_{i}{ }^{N}$ in $(2.1)$ are determined by the boundary condition of zero traction on the surface $x_{3}=0$. The resulting consistency condition gives the secular equation determining $s_{1}$ :

$$
-4 s_{3}{ }^{1} s_{3}{ }^{2}=s_{1}{ }^{2}\left(2-\rho / \mu s_{1}{ }^{2}\right)^{2} .
$$

If we write

$$
c=\rho / \mu s_{1}^{2},
$$

then squaring (2.4) and using (2.2) we find

$$
c^{3}-8 c^{2}+(24-16 \mu /[\lambda+2 \mu]) c-16(1-\mu /[\lambda+2 \mu])=0 .
$$

This equation determines $c$ and hence $s_{1}$. But not all solutions of (2.6) are admissible. Spurious solutions have been introduced through squaring (2.4). In addition, the wave must decay with depth and with distance along the surface. Thus, as in [1], a root of (2.6) is said to be admissible if corresponding to it

(i) $s_{1}{ }^{+}>0, s_{1}^{-} \geq 0$,

(ii) $s_{3}{ }^{1}<0, s_{3}{ }^{2}<0$,

(iii) the secular equation (2.4) is satisfied.

For the elastic case, Hayes and Rivlin [2] have shown that there is only one admissible root. However, for the viscoelastic case, it was shown in [1] that for particular values of the moduli $\lambda, \mu$ there may be either one or two roots.

These moduli $\lambda$ and $\mu$ are frequency-dependent, with the nature of this dependence unknown until the constitutive equation is specified in detail. From the general form of the constitutive equation [1, Eqs. (2.6), (2.7)], we find integrating by parts, that

$$
\begin{gathered}
\mu\left(\delta_{i k} \delta_{j m}+\delta_{j k} \delta_{i m}\right)+\lambda \delta_{k m} \delta_{i j}=c_{i j k m}+\int_{-\infty}^{t} f_{i j k m}\left(t-t^{\prime}\right) \exp i \omega\left(t-t^{\prime}\right) d t^{\prime} \\
=c_{i j k m}-i \omega \int_{-\infty}^{t} F_{i j k m}\left(t-t^{\prime}\right) \exp i \omega\left(t-t^{\prime}\right) d t^{\prime},
\end{gathered}
$$

where the constants $C_{i j k m}$ and the functions $f_{i j k m}$ determine the response of the material:

$$
C_{i j k m}=c_{i j k m}+F_{i j k m}(0), F_{i j k m}^{\prime}(t)=f_{i j k m}(t) .
$$


In Sec. 4 we consider two special constitutive equations. For the Kelvin-Voigt material

$$
\begin{aligned}
& C_{i j k m}=\hat{\mu}\left(\delta_{i k} \delta_{j m}+\delta_{j k} \delta_{i m}\right)+\hat{\lambda} \delta_{i j} \delta_{k m}, \\
& F_{i j k m}=\delta(t)\left[\hat{\mu} \tau\left(\delta_{i k} \delta_{i m}+\delta_{j k} \delta_{i m}-\frac{2}{3} \delta_{i j} \delta_{k m}\right)+\left(\hat{\lambda}+\frac{2}{3} \hat{\mu}\right) \theta \delta_{i j} \delta_{k m}\right],
\end{aligned}
$$

where $\hat{\lambda}, \hat{\mu}$ are constant moduli and $\tau, \theta$ are constant relaxation times. For the Maxwell material $C_{i j k m}=0$ and

$F_{i j k m}=\hat{\mu} \exp (-t / \tau)\left(\delta_{i k} \delta_{j m}+\delta_{j k} \delta_{i m}-\frac{2}{3} \delta_{i j} \delta_{k m}\right)+\left(\hat{\lambda}+\frac{2}{3} \hat{\mu}\right) \exp (-t / \theta) \delta_{i j} \delta_{k m}$.

From these constitutive equations it follows that the complex Lamé moduli appearing in (2.2)-(2.6) are given by

$$
\begin{array}{cc}
\mu=\hat{\mu}(1-i \omega \tau), & \text { Kelvin-Voigt } \\
3 \lambda+2 \mu=(3 \hat{\lambda}+2 \hat{\mu})(1-i \omega \theta), & \\
\mu=-i \omega \tau \hat{\mu} /(1-i \omega \tau), & \text { Maxwell } \\
3 \lambda+2 \mu=-i \omega \theta(3 \hat{\lambda}+2 \hat{\mu}) /(1-i \omega \theta), &
\end{array}
$$

We note here that, in addition to the works cited in [1], Borcherdt [4] has also studied surface waves on viscoelastic materials. Some of the results reported in [1] on the elliptical particle paths were anticipated by Borcherdt, but his discussion is less detailed.

3. The number of admissible roots. It was found in [1] that there may be more than one solution of (2.6) satisfying the admissibility conditions (2.7). At various values of $\lambda$ and $\mu$ the number of admissible roots changed. In this section we investigate these critical values of the moduli.

a. Conditions for critical values. Eq. (2.6) was solved numerically for given values of $\lambda$, $\mu$. For each of the roots, $s_{1}{ }^{2}$ and $s_{3}{ }^{112}$ were calculated from (2.2). It was always found possible to choose $s_{1}$ to satisfy (2.7) (this presumably follows from the thermodynamic restrictions (2.3), although we have not proved this). Moreover, $s_{3}{ }^{(1)}$ can always be chosen to satisfy (2.7), provided $\left(s_{3}{ }^{11}\right)^{2}$ is not purely real and positive. $s_{3}{ }^{12}$ was then calculated from (2.4). The root was considered admissible if the resulting value for ${S_{3}}^{2}{ }^{2)}$ satisfied (2.7).

It is clear from this procedure that as $\lambda$ and $\mu$ are varied, a root becomes inadmissible only if either

$$
s_{3}^{(1)-}=0
$$

or

$$
s_{3}^{22-}=0 .
$$

From (2.2) and (2.5), (3.1) and (3.2) imply either

$$
\left(\frac{c-1}{c \mu}\right)^{-}=0
$$

or

$$
\left(\frac{c \mu /[\lambda+2 \mu]-1}{c \mu}\right)^{-}=0 .
$$

It is possible to substitute from either (3.3) or (3.4) into (2.6) and then to eliminate $c$ to give a relationship that must be satisfied when a root becomes inadmissible. This approach is algebraically complicated and has the disadvantage that it uses (2.6) rather than the original secular equation (2.4). Thus spurious critical values arise, corresponding to 


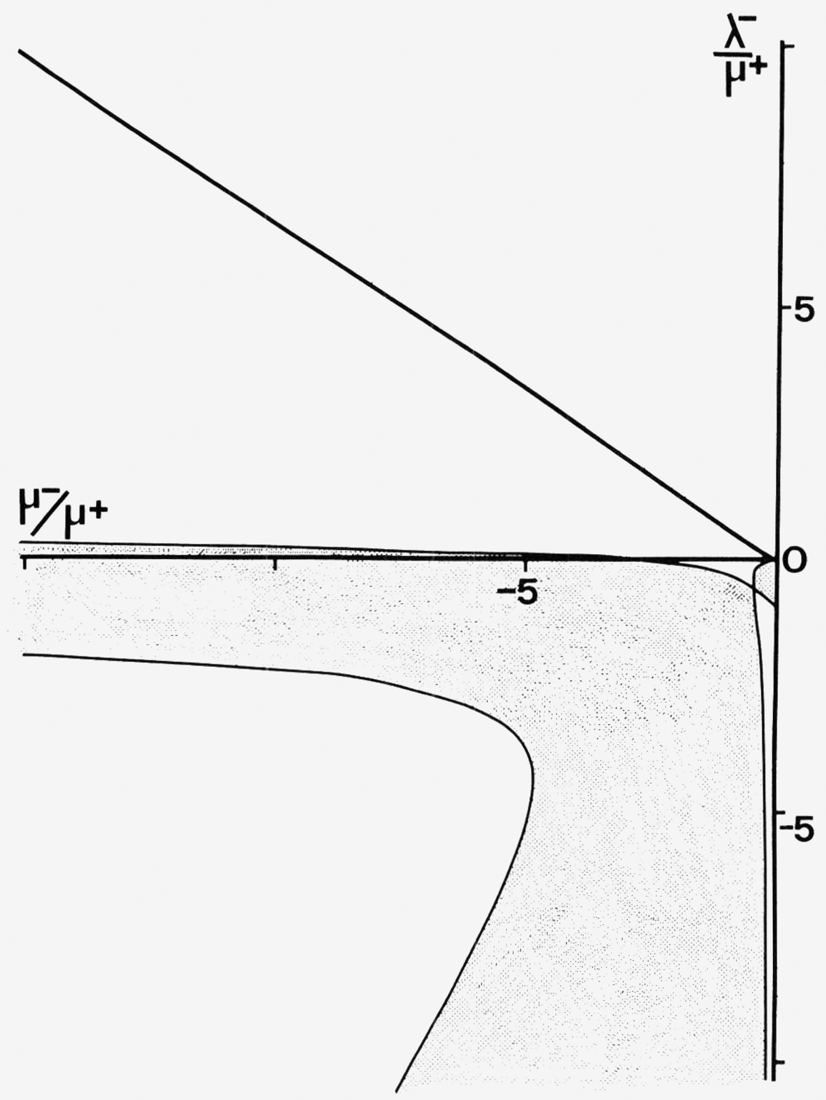

FIG. 1. $\lambda^{+} / \mu^{+}=-\frac{2}{3}$. Critical curves as functions of $\mu^{-} / \mu^{+}$and $\lambda^{-} / \mu^{+}$. Regions with two surface waves are shaded. Thermodynamically allowable values of $\lambda^{-}$and $\mu^{-}$satisfy $3 \lambda^{-}+2 \mu^{-}<0$.

spurious roots of (2.6). Nevertheless, this procedure has been carried out for the two cases considered in detail in [1] (the incompressible material and the material with $\lambda^{+}=\mu^{+}$and small viscosity), in both cases confirming the critical values found before.

b. Asymptotic behavior. An algebraic analysis is useful, however, for considering the asymptotic behavior for large $\mu^{-}$or $\lambda^{-}$. If $\left|\lambda^{-}\right| \gg \lambda^{+},\left|\mu^{-}\right| \gg \mu^{+}$, then it follows from (3.4) that

$$
c^{-1} \sim \mu^{-} /\left(\lambda^{-}+2 \mu^{-}\right)+k i,
$$

for some real $k$. This expression is substituted into the limiting form of (2.6) for large $\left|\mu^{-}\right|$, $\left|\lambda^{-}\right|$. The real and imaginary parts of the resulting equation give two expressions for $k^{2}$ which are consistent if either

$$
\lambda^{-} / \mu^{-}=1.8219
$$

or

$$
\lambda^{-} / \mu^{-}=0, \quad c \sim 2 .
$$

But, eliminating $\mu /(\lambda+2 \mu)$ between (2.2) and (2.6), we have

$$
\left[s_{3}{ }^{(2)}\right]^{2}=\rho(c-2)^{4} / 16 \mu c(c-1),
$$




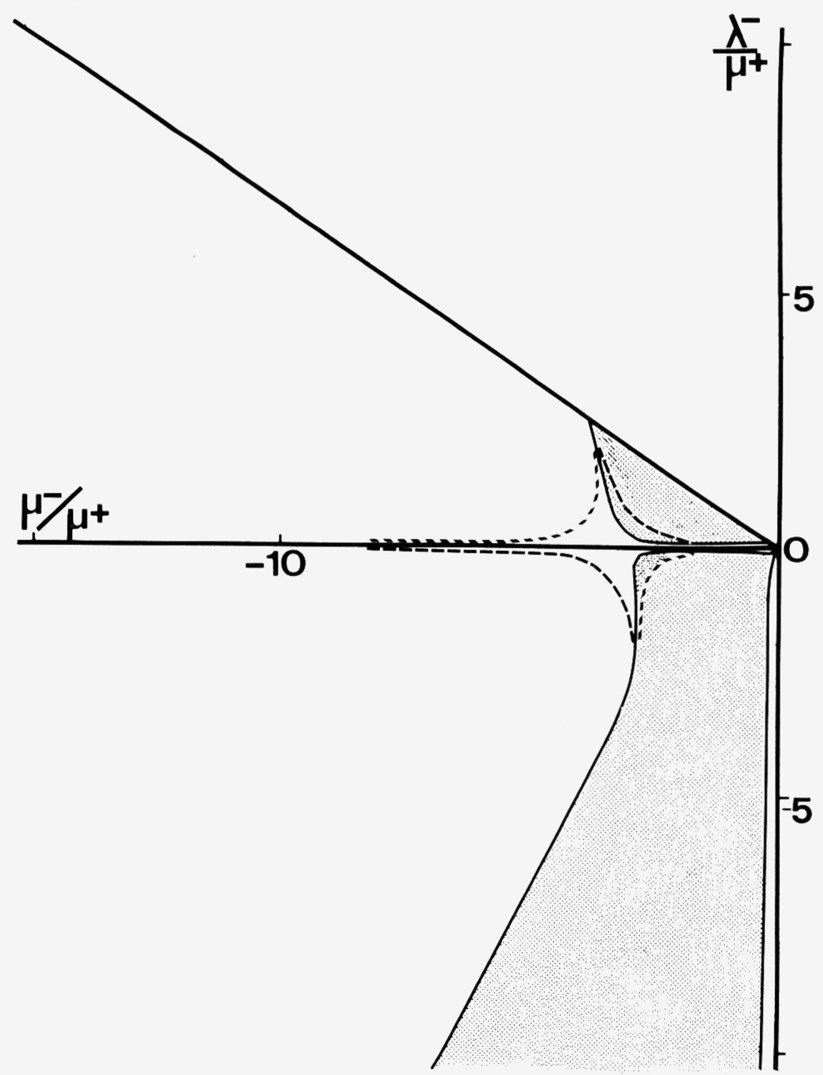

Fig. 2. As for Fig. 1, with $\lambda^{+} / \mu^{+}=0$. Also shown are the critical curves for $\lambda^{+} / \mu^{+}=0.03---\mu^{+}$ $\lambda^{+} / \mu^{+}=-0.03-----$

and from $(2.6)$

$$
2(c-2)\left(c^{2}-6 c+4\right) \mu=-\lambda\left(c^{3}-8 c^{2}+24 c-16\right) .
$$

Eliminating the factor $(c-2)$ between (3.8) and (3.9), we find

$$
\frac{\left[s_{3}{ }^{(2)}\right]^{2}}{\rho}=\frac{\lambda^{4}}{\mu^{5} c(c-1)}\left\{\frac{c^{3}-8 c^{2}+24 c-16}{4\left(c^{2}-6 c+4\right)}\right\}^{4} .
$$

As $\mu^{-} \rightarrow-\infty$, corresponding to the asymptotic values $(3.7),\left[s_{3}{ }^{(2)}\right]^{2}$ is purely real only if $\lambda^{4}$ is negative imaginary in (3.10). Hence the asymptotic values of $\lambda^{-}$corresponding to (3.7) are

$$
\begin{array}{cc}
\lambda^{-} \sim(\sqrt{ } 2+1) \lambda^{+}, & \mu^{-} \rightarrow-\infty, \\
\lambda^{-} \sim-(\sqrt{2}-1) \lambda^{+}, & \mu^{-} \rightarrow-\infty .
\end{array}
$$

In a similar fashion the asymptotic behavior for inadmissible $s_{3}^{(1)}$ can be found. The only asymptote which is not spurious is given by

$$
\mu^{-}=-0.159 \mu^{+}, \lambda^{-} \rightarrow-\infty .
$$

This is recognized as the critical line found for an incompressible material [1, Eq. (6.8)]. 


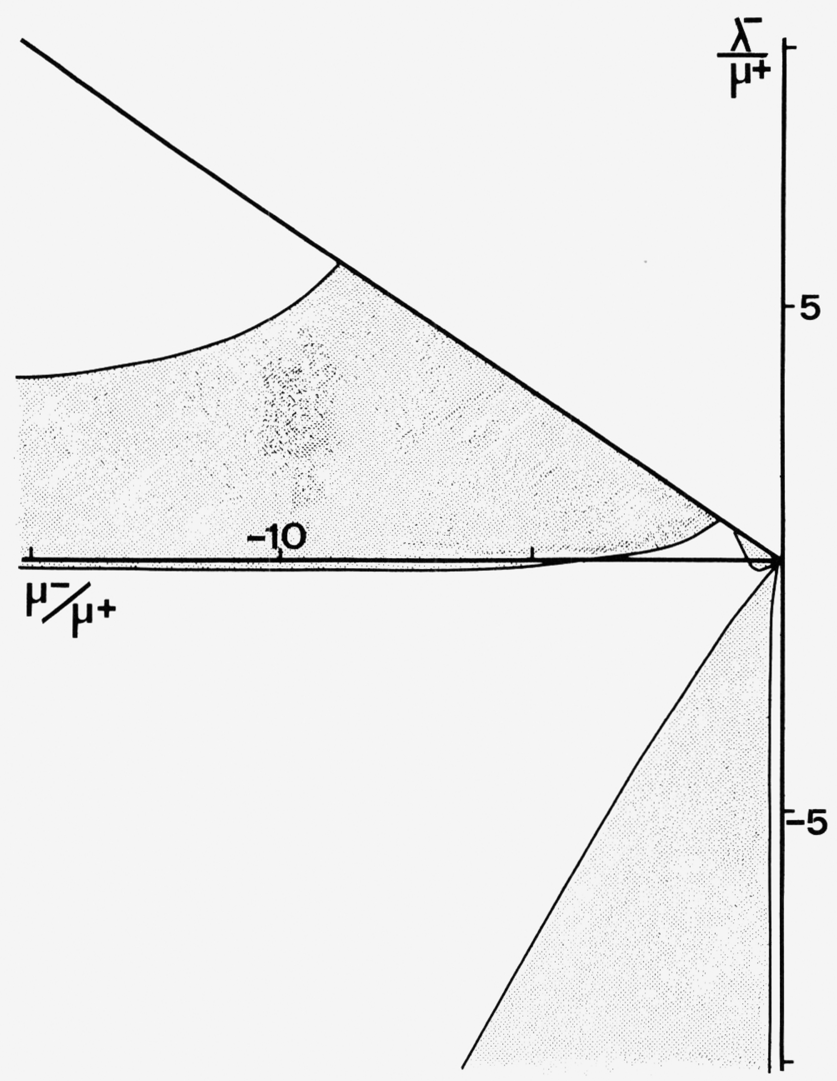

FIG. 3. As for Fig. 1, with $\lambda^{+} / \mu^{+}=1$.

Thus the asymptotic behavior of the critical values for large $\lambda^{-}$and $\mu^{-}$is given by (3.6), (3.11) and (3.12).

c. Numerical results. In general, however, the critical values must be found by computation. In Figs. 1-5 the critical values of $\lambda^{-} / \mu^{+}$are shown as functions of $\mu^{-} / \mu^{+}$for $\lambda^{+} / \mu^{+}$ taking the fixed values $-\frac{2}{3}, 0,1,2,5$. The regions with two admissible roots are shaded on the figures.

It is clear from these figures that it is not just in small isolated regions that there are two admissible roots. Indeed, there seems no a priori reason why, for a given material at a given frequency, the calculated number of roots should be one rather than two.

The most constant feature of the figures is the curve corresponding to the asymptote (3.12). As $\lambda^{+} / \mu^{+}$increases, this curve tends towards the straight line $\mu^{-}=-0.159 \mu^{+}$found for an incompressible material in [1]. The other curves vary more with $\lambda^{+} / \mu^{+}$. For $\lambda^{+}=0$, the curves coalesce along the $\mu^{-}$-axis, so that the asymptotes (3.11) do not exist. But for $\lambda^{+} / \mu^{+}$small these asymptotes do exist and are very close together. This is shown in Fig. 2 explicitly for the cases $\lambda^{+} / \mu^{+}= \pm 0.03$. As $\lambda^{+} / \mu^{+}$increases beyond the value 1 the picture becomes more predictable, with the curves corresponding to the asymptotes (3.11) moving out from the origin proportionally to $\lambda^{+} / \mu^{+}$.

We have found no values of $\lambda$ and $\mu$ for which there are more than two roots. 


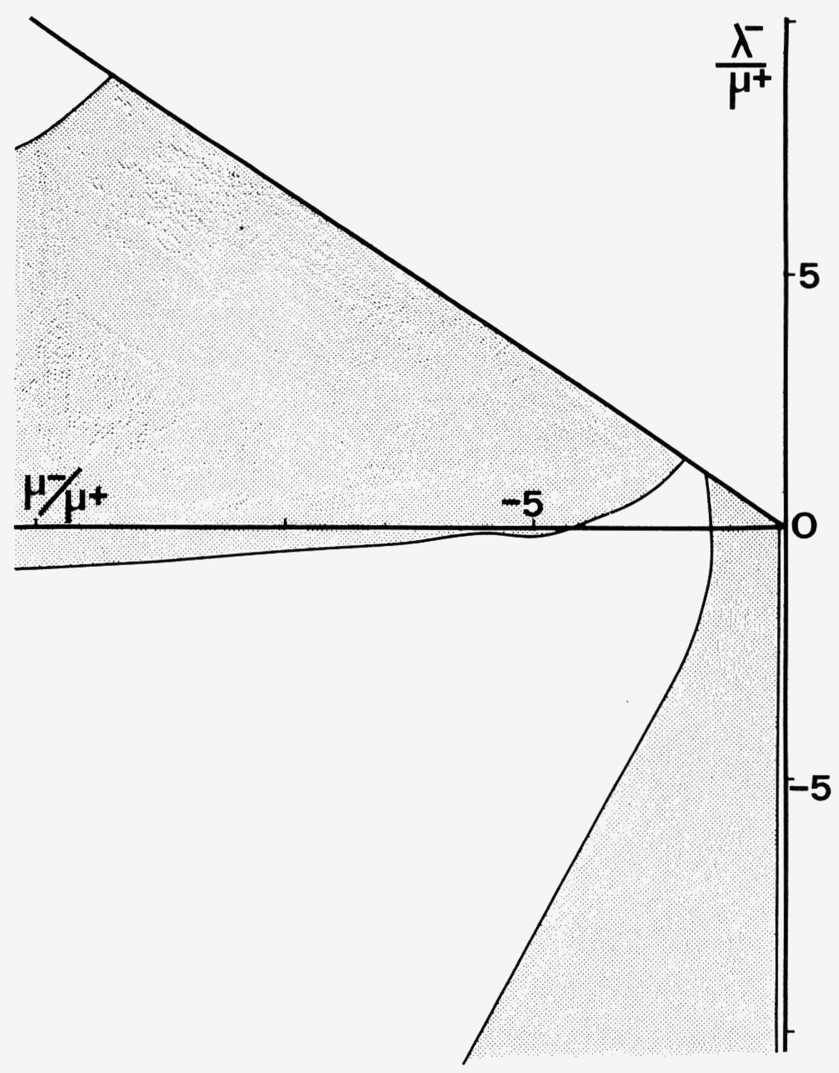

FIG. 4. As for Fig. 1, with $\lambda^{+} / \mu^{+}=2$.

4. Particular models: Kelvin-Voigt and Maxwell materials. In this section we examine the implications of the general results given in the figures for two theoretical models.

a. Kelvin-Voigt material. If the material is a Kelvin-Voigt material defined by (2.9) and (2.11) then $\lambda^{+}=\hat{\lambda}, \mu^{+}=\hat{\mu}, \mu^{-}=-\hat{\mu} \omega \tau, \lambda^{-}=-\hat{\lambda} \omega \theta+\frac{2}{3} \hat{\mu} \omega(\tau-\theta)$. For given values of $\hat{\lambda}$, $\hat{\mu}, \tau, \theta$ the number of roots can be read off directly from the figures. The line $\mu^{-} / \mu^{+}=-\omega \tau$, $\lambda^{-} / \mu^{+}=-\omega \theta \hat{\lambda} / \hat{\mu}+\frac{2}{3} \omega(\tau-\theta)$ (where $\omega$ is a parameter) is drawn on the figure corresponding to the particular value of $\lambda^{+} / \mu^{+}=\hat{\lambda} / \hat{\mu}$. The values of $\omega$ at which the number of roots changes can then be determined. For example, if $\hat{\lambda}=\hat{\mu}$ and $\tau=5 \theta$, then from Fig. 3 we find

$$
\begin{aligned}
& 0<\omega \tau<0.8, \quad 2 \text { roots } \\
& 0.8 \leq \omega \tau \leq 1.7, \quad 1 \text { root } \\
& 1.7<\omega \tau<12.1 \quad 2 \text { roots } \\
& 12.1 \leq \omega \tau, \quad 1 \text { root. }
\end{aligned}
$$

However, if $\hat{\lambda}=\hat{\mu}$, then, whatever the frequency, there is only one root if $\tau=\theta$ and always two roots if $8 \tau=5 \theta$.

b. Maxwell material. For the Maxwell material, defined by (2.10) and (2.12), the 


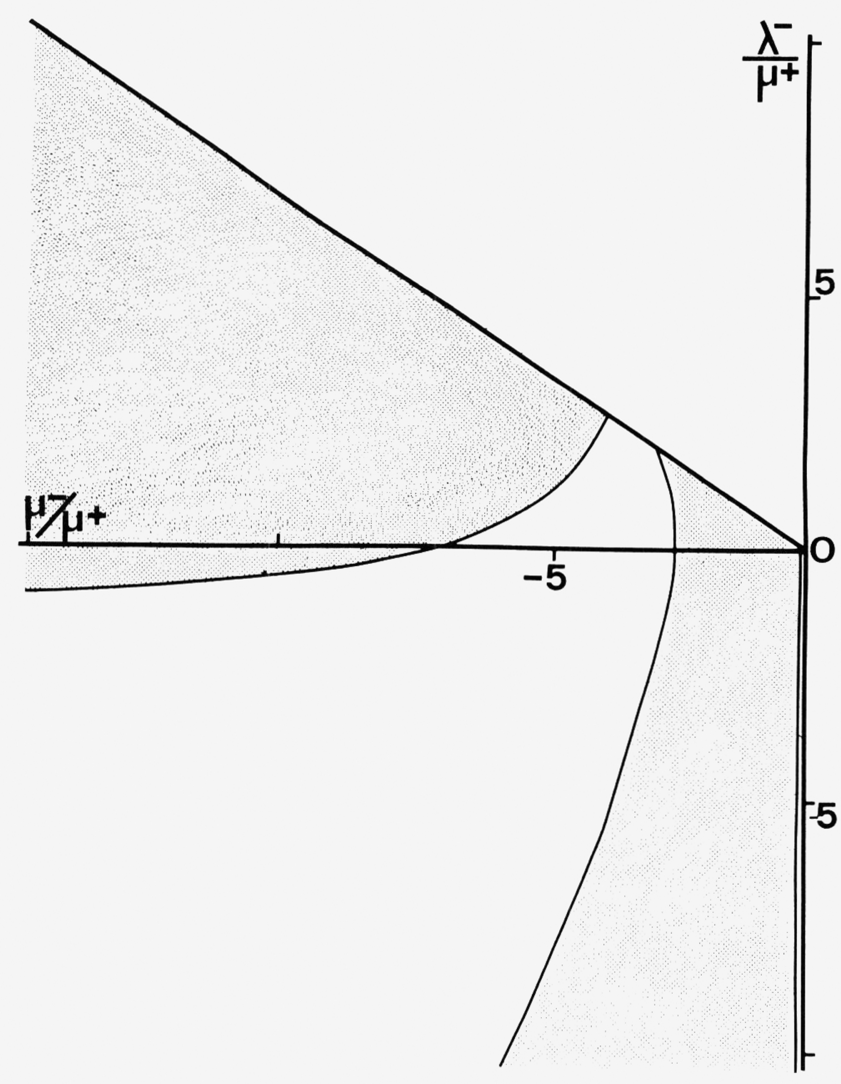

FIG. 5. As for Fig. 1, with $\lambda^{+} / \mu^{+}=5$.

analysis is more complicated. From (2.12) we have

$$
\begin{aligned}
\mu^{-} / \mu^{+} & =-1 / \omega \tau \\
\frac{3 \lambda^{+}+2 \mu^{+}}{\mu^{+}} & =\left(\frac{3 \hat{\lambda}+2 \hat{\mu}}{\hat{\mu}}\right) \frac{\left(1+\omega^{2} \tau^{2}\right) \theta^{2}}{\left(1+\omega^{2} \theta^{2}\right) \tau^{2}} \\
\frac{3 \lambda^{-}+2 \mu^{-}}{\mu^{+}} & =-\frac{\theta}{\omega \tau^{2}} \frac{(3 \hat{\lambda}+2 \hat{\mu})}{\hat{\mu}} \frac{\left(1+\omega^{2} \tau^{2}\right)}{\left(1+\omega^{2} \theta^{2}\right)} .
\end{aligned}
$$

It follows that

$$
\frac{3 \lambda^{-}+2 \mu^{-}}{\mu^{-}}=\frac{\tau}{\theta}\left(\frac{3 \lambda^{+}+2 \mu^{+}}{\mu^{+}}\right) .
$$

A simple case is $\theta=\tau$. Then $\lambda^{-} / \mu^{-}=\lambda^{+} / \mu^{+}=\hat{\lambda} / \hat{\mu}$. For this case it is found from the figures that there is only one root if $\hat{\lambda} / \hat{\mu}=-\frac{2}{3}, 0$ or 1 , but for $\hat{\lambda} / \hat{\mu} \geq 2$ there is always a critical frequency corresponding to the root (2.12). Thus, to a very good approximation, if $\hat{\lambda} / \hat{\mu} \geq 2$

$$
\begin{array}{ll}
0 \leq \omega \tau<1 / 0.159=12.58, & \text { two roots, } \\
12.58 \leq \omega \tau, & \text { one root. }
\end{array}
$$


For the general case it is difficult to deduce accurately the critical frequencies from the general results given in the figures. One method of proceding is to calculate from (4.3) the ratio of $\lambda^{-} / \mu^{-}$for given $\theta / \tau, \lambda^{+} / \mu^{+}$and to read off from the figures the corresponding critical values of $\lambda^{-} / \mu^{+}$and $\mu^{-} / \mu^{+}$. The resulting critical ratio $\hat{\lambda} / \hat{\mu}$ and $\omega \tau$ can then be found from (4.2). Plotting these values on a graph allows for interpolation for other values of $\theta / \tau$. This procedure is highly inaccurate, and independent calculation is preferable.

These results for the Kelvin-Voigt and Maxwell models indicate that the number of possible surface waves depends on the frequency, in general. For a particular constitutive equation calculation will usually be necessary to determine the critical values of the frequency at which the number of roots changes.

5. Experimental values of the material parameters. In this section we present experimental data indicating that two surface waves are possible on real materials. We consider rocks and solid polymers.

a. Rocks. We consider first the data from rocks compiled from various sources by White [5]. Since values of $\lambda^{+} / \mu^{+}, \lambda^{-} / \mu^{+}, \mu^{-} / \mu^{+}$, are needed, the number of surface waves can be determined only for those rocks for which White gives the wave speed and loss parameter for both longitudinal and transverse body waves. In Table 1 we list these rocks, the values of $\lambda^{+} / \mu^{+}, \lambda^{-} / \mu^{+}, \mu^{-} / \mu^{+}$computed from White's data, the corresponding angular frequency $\omega$, and the number of surface waves. It is seen that for granite over the frequency range $10^{2}-10^{5}$ c.p.s. two surface waves are possible.

b. Solid polymers. For viscoelastic solid polymers there is a wealth of experimental data given by Ferry [3]. Most solid polymers are "soft", according to Ferry, in the sense that they are much more easily sheared than compressed. This is confirmed by the values of Poisson's ratio given by Haward [6] for solid polymers, from which we calculate that $\lambda^{+} / \mu^{+}=1.9$ for polystyrene and polymethyl methacrylate, 3.2 for polyethelyne and 49 for rubber. Thus all these solid polymers behave almost like incompressible materials. For incompressible materials it was shown in [1] that two surface waves are possible if $\mu^{-} / \mu^{+}$ $<-0.159$. In Ferry's notation $-\mu^{-} / \mu^{+}=\tan \delta$, the loss tangent. A more careful analysis, based on the experimental data and the figures given in this paper, confirms that the only relevant critical value for the loss tangent is $\tan \delta=0.159$ (i.e. $\log \tan \delta=-1.84$ ). If $\tan \delta$ $>0.159$ there are two surface waves; otherwise there is one surface wave. It is now possible to read off the critical values of frequency for various solid polymers from the figures given by Ferry (referred to by their numbers in [3]). From his Fig. 2-8 it is clear that for a solid polymer there are typically two possible surface waves. But it also appears possible that at low-frequencies ( $\omega \sim 1$ c.p.s.) there may be only one wave for rubber, and at high-

TABLE 1. The number of surface waves $n$ for various rock types (data computed from White [5, Table 3.1]).

\begin{tabular}{|c|c|c|c|c|c|}
\hline Rock type & $\begin{array}{l}\text { frequency } \\
\text { c.p.s. }\end{array}$ & $\lambda^{-} / \mu^{+}$ & $-\lambda / \mu$ & $-\mu^{-} / \mu^{+}$ & $n$ \\
\hline Granite: Sample 3 & $(30-180) \times 10^{3}$ & .776 & .063 & .035 & 2 \\
\hline Sample 4 & $(30-180) \times 10^{3}$ & .663 & .027 & .016 & 2 \\
\hline Quincy & $140-4,500$ & .342 & .0067 & .006 & 2 \\
\hline Limestone: Solenhofen & $(3-15) \times 10^{6}$ & 1.68 & .022 & .005 & 1 \\
\hline Sample I-1 & $(3-15) \times 10^{6}$ & 1.63 & .017 & .0024 & 1 \\
\hline Chalk: Chislehurst & 600 & 1.44 & .0014 & .0005 & 1 \\
\hline Shale: Pierre & $\left\{\begin{array}{l}50-450 \\
20-125\end{array}\right\}$ & 5.43 & .024 & .103 & 1 \\
\hline
\end{tabular}


frequencies ( $\omega \sim 10^{5}$ c.p.s. ) there may be only one wave for polyethelyne. This is confirmed by the more detailed results given in his Fig. 12-9 which show that at both high and low frequencies there may be just one wave for rubber, but that generally there are two. His Fig. 15-1 indicates that there is only one wave for polystyrene, but that there are two waves for polymethyl methacrylate except possibly at high frequency (although this seems to be contradicted by the data given in Fig. 15-8 which imply that there is only one wave for polymethyl methacrylate, whatever the frequency). Finally we note that the loss tangent varies considerably with temperature (see, for example, his Fig. 16-20). Thus the number of possible surface waves will depend on temperature as well as frequency.

There is therefore considerable evidence that two surface waves are possible on many solid polymers. Typically $\log \tan \delta$ varies between -1.2 and -0.4 , except at extreme values of the frequency; i.e., $-\mu^{-} / \mu^{+} \approx 0.3-0.7$. It was shown in [1, Eqs. (6.10), (6.11)] that for $\mu^{-} / \mu^{+}=-0.5$ the speeds of the two waves along the surface are $1.04\left(\mu^{+} / \rho\right)^{1 / 2}$ and 2.48 $\left(\mu^{+} / \rho\right)^{1 / 2}$ and the rate of exponential decay with distance is $0.23 \omega\left(\rho / \mu^{+}\right)^{1 / 2}$ per unit length for both waves.

6. Conclusions. The numerical results presented in this paper suggest that there is no a priori reason why for a given viscoelastic material at a given frequency the number of possible surface waves should be one rather than two. The results for the Kelvin-Voigt and Maxwell materials indicate that the number of waves varies with the frequency and does not just depend on the material parameters. This will presumably be true for more realistic models of viscoelastic behavior, such as the standard linear model. However, it is clear from Sec. 4 that further computation will generally be needed for any specific constitutive equation.

The experimental data for rocks reviewed in Sec. 5 show that two surface waves are possible on granite. However, for materials with small viscous terms (such as granite) it was shown in [1] that one of the waves decays very slowly with depth and may be of less importance than the other wave.

For solid polymers the experimental data show that there are typically two surface waves, both with similar properties.

\section{REFERENCES}

[1] P. K. Currie, M. A. Hayes and P. M. O'Leary, Viscoelastic Rayleigh waves, Quart. Appl. Math. 35, 35-53 (1977)

[2] M. A. Hayes and R. S. Rivlin, A note on the secular equation for Rayleigh waves, ZAMP 13, 80-83 (1962)

[3] J. D. Ferry, Viscoelastic properties of polymers, 2nd edition, Wiley, New York, 1970

[4] R. D. Borcherdt, Rayleigh-type surface wave on a linear viscoelastic half-space, J. Acoust. Soc. Amer. 54, $1651-1653$ (1973) 55, 13-15 (1974).

[5] J. E. White, Seismic waves: radiation, transmission and attenuation, McGraw-Hill, New York, 1965

[6] R. N. Haward, Introduction, in The physics of glassy polymers, ed. R. N. Haward, Applied Science, London, 1973 